\title{
Huge avenues of opportunities (with some potholes) opened by the very small things
}

\author{
Volume I Issue I - 2014 \\ Carlos Luna Criado \\ IFaculty of Physical and Mathematical Sciences, Autonomous \\ University of Nuevo Leon, Mexico

\begin{abstract}
Correspondence: Carlos Luna Criado, Research Centre in Physical and Mathematical Sciences/ Faculty of Physical and AV University S/N, Mexico, Tel I-9|8-208-0288, Fax |-9|8-5।2468I Email carlos.junacd@uanl.edu.mx
\end{abstract} \\ Mathematical Sciences, Autonomous University of Nuevo Leon,
}

\section{Editorial}

In its incessant exploration of the cosmos into the cosmos, crossing the dimensionality barrier, science is living a unique moment with an unstoppable knowledge growth ranging from the subtle details of the immensity to the vastness of the very small. In the last aspect, progress is now incredible extremely rapid, and today, shape the matter at nanoscale to prepare engineered low dimensional systems (thin films, elongated nanostructures and nanocrystals and quantum dots) is an everyday reality common throughout the world. Nanoscopic objects with an increasing (and sometimes funny) variety of sizes and forms, including cubes. ${ }^{1}$ ellipsoids. ${ }^{2}$ cylinders. ${ }^{3}$ multisegmented cylinders. ${ }^{4}$ tubes. $^{5}$ tubes in tubes. ${ }^{6}$ dendrites. ${ }^{7}$ and flower-like. ${ }^{8}$ peanut-like ${ }^{9}$ and popcorn-like structures. ${ }^{10}$ have been successfully obtained and studied, providing new insights on the physicochemical properties of the matter with an invaluable technological potential. Particularly, nanomaterials, through their interactions with the electromagnetic radiation, electrons, molecules, exciting electric or magnetic fields and/or tensile stresses, allow us to intervene on physical processes at the nanometric (or even atomic) scale, providing new principles to develop innovative sensors, nano-electro-mechanical systems (NEMS), magnetic random access memories (MRAMs) and revolutionary electronic and photonic devices. In this manner, during the last decades and to our astonishment, we have witnessed the detection of single molecules. ${ }^{11}$ the measure of temperature and $\mathrm{pH}$ fluctuations at the nanoscale. ${ }^{12}$ the preparation of nanomaterials with Young's modulus several times than that of the diamond. ${ }^{5}$ the finding of new sources of magnetic anisotropy related to the low dimensionality. ${ }^{13}$ and its use to shift the superparamagnetic limit for the magnetic recording media. ${ }^{14}$ the spin-dependent trapping of electrons.$^{15}$ the efficient transport of excitons to design novel light sources of many emission-colors. ${ }^{16}$ and the amazing electron transport in Graphene similar than relativistic particles with zero rest mass and with an "effective speed of light" of $106 \mathrm{~ms}-1 .{ }^{17}$ among other many surprising events.

Equally impressive are the great advances achieved with the exploitation of the uniqueness potential of nanomaterials in the biomedical area, which results in the fascinating and rapidly evolving field of nanomedicine. In this regard, the astonishing physicochemical properties of nanomaterials along with their extremely reduced size, which have at least one of its dimensions hundred or thousand times smaller than the diameter of a red blood cell, have allowed proposing new promising approaches for solving clinical problems that, have not been successfully resolved with conventional techniques. For instance, in suitable conditions, the use of very fine colloidal nanoparticles as nanoscopic vehicles of therapeutic agents (drugs, genes, and radioactive isotopes) improve the cancer therapies increasing the pharmacokinetic profiles, the bioavailability and the tissue distribution of such agents in comparison with the obtained with other formulations. ${ }^{18}$ On the other hand, new tactical solutions
Received: October 01, 2014 | Published: October 10, 2014

to achieve the desired site-specific drug delivery based on the use of functionalized colloidal nanoplatforms are now being actively investigated. Such strategies are based on ligand-receptor or antibodyantigen interactions..$^{19-21}$ and/or the use of superparamagnetic colloidal nanoparticles guided by exciting magnetic field gradients. ${ }^{22}$ In addition, these last nanoparticles can perform other tasks after reaching a damaged tissue or organ. For example, given their superparamagnetic behavior, they are excellent contrast agents for MRI (standard and functional). ${ }^{23}$ In fact, several commercial MRI contrast agents (e.g. Endorem ${ }^{\circledR} /$ Feridex IV ${ }$, Gastro MARK ${ }^{\circledR}$ and Resovist ${ }^{\circledR}$ ) are colloidal suspensions of functionalized super paramagnetic iron oxide nanoparticles (SPIONs). Furthermore, SPIONs also can serve as tiny heat radiation sources $\left(42-56^{\circ} \mathrm{C}\right)$ in the presence of an alternating magnetic field (typically with amplitudes of 1 to $50 \mathrm{kA} / \mathrm{m}$ and frequencies of $1 \mathrm{kHz}$ to $1 \mathrm{MHz}$ ) by means of Neel and/or Brownian relaxations. $^{24}$

Metallic noble nanoparticles are also key nanosystems with surprising implications in the life sciences. For instance, given their tunable plasmonic. ${ }^{25}$ and surface-enhanced Raman scattering (SERS).$^{26}$ properties, noble metal nanoparticles can be used as optical nanoantenna for the ultra-high sensitive detection of biomolecules (DNA, proteins, prions, etc) ${ }^{27-29}$ in different complex media such as serum and blood. ${ }^{29} \mathrm{Also}$, they have been used as photo-adsorbing agents in photo-thermal therapies. ${ }^{30}$ On the other hand, silver nanoparticles display potent and broad-spectrum antibacterial. ${ }^{7,31}$ antifungal. ${ }^{32}$ and virucidal. ${ }^{33}$ activities that have been widely exploited in the disinfection of aliments, water and medical instruments, and which could play an essential role in the prevention of epidemics caused by the progressive resistance of pathogens to the drugs. ${ }^{31}$ Also, Ag NPs can display positive effects in the healing and regeneration of tissues through their antimicrobial activity, the reduction of the inflammation processes and the modulation of fibrogenic cytokines..$^{34,35}$ and Ag NPs 
deposited on a substrate can serve as favorable anchoring sites to promote the development of neurites in the neuronal growth. ${ }^{36}$

All these spectacular advances have generated an increasing enthusiasm about the future of the application of engineered nanomaterials in medicine; however important challenges and difficulties must be urgently addressed. For instance, the penetration depth and drug intra-tumoral diffusion using nanoscopic drug vectors must be improved to achieve an actually successful therapy in clinical applications. Furthermore, although nanomaterials don't provoke massive death of human cells as it has been observed for bacteria exposed to certain nanoparticles, very fine nanomaterials can significantly affect to their health inhibiting their metabolism and producing DNA damage. ${ }^{37}$ Also, nanotoxicology studies in invivo systems have yielded disturbing results. For instance, researches of aquatic systems treated with nanomaterials have indicated a concentration-dependent increase of biological dysfunctions, including a reduction of the fertilization and reproduction success and mortality and hatching delay in fish embryos. ${ }^{38,39}$

Therefore, we are on the verge of a new technological revolution where awesome applications are emerging and whose range is hard to imagine. They should radically improve various aspects of our quality of life. However, to make this a reality without jeopardizing human health and the environment, further investigations are pressingly required to fully understand the mechanisms involved in the bioactivity of the nanomaterials and their dependences to achieve a sustainable exploitation of the spectacular technological and biomedical potential of the nanomaterials.

\section{Acknowledgment}

None.

\section{Conflict of interest}

None.

\section{References}

1. Sun Y, Xia Y Shape-controlled synthesis of gold and silver nanoparticles. Science. 2002;298(5601):2176-2179.

2. Mendoza-Resendez R, Luna C, Barriga-Castro ED et al Control of crystallite orientation and size in $\mathrm{Fe}$ and $\mathrm{FeCo}$ nanoneedles. Nanotechnology. 2012;23(22):225601.

3. Duan X, Huang Y, Cui $\mathrm{Y}$ et al. Indium phosphide nanowires as building blocks for nanoscale electronic and optoelectronic devices. Nature. 2001;409(6816):66-69.

4. Prida VM, Garcia J, Iglesias L et al. Electroplating and magneto structural characterization of multisegmented Co54Ni46/Co85Ni15 nanowires from single electrochemical bath in anodic alumina templates. Nanoscale Res Lett. 2013;8(1):263.

5. Treacy MM, Ebbesen TW, Gibson JM Exceptionally High Young's Modulus Observed for Individual Carbon Nanotubes. Nature. 1996;381(6584):678-680

6. Jia CJ, Sun LD, Yan ZG et al. Iron oxide tube-in-tube nanostructures. The Journal of Physical Chemistry. 2007;111(35):13022-13027.

7. Mendoza-Resendez R, Gomez-Trevino A, Barriga-Castro ED et al Synthesis of antibacterial silver-based nanodisks and dendritic structures mediated by royal jelly. RSC Advances. 2014;4(4):1650-1658.

8. Zhang H, Yang D, Ma X, Ji Y, Xu J, et al. Synthesis of flowerlike $\mathrm{ZnO}$ nanostructures by an organic-free hydrothermal process. Nanotechnology. 2004;15(5):622.
9. Wang M, Huang QL, Zhong HX et al. Formation of YF3 nanocrystals and their self-assembly into hollow peanut-like structures. Crystal Growth \& Design. 2007;7(10):2106-2111.

10. Luna C, Barriga-Castro ED, Mendoza-Resendez R The effects of aging time on the size, morphology, oriented attachment and magnetic behavior of hematite nanocrystals synthesized by forced hydrolysis of FeIII solutions. Acta Materialia. 2014;66:405-413.

11. Kneipp K, Wang Y, Kneipp H et al. Single molecule detection using surface-enhanced Raman scattering (SERS). Physical Review Letters. 1997;78:1667-1670.

12. Lee J, Govorov AO, Kotov NA Nanoparticle assemblies with molecular springs: A nanoscale thermometer. Angew Chem Int EdEngl. 2005;44:7439-7442.

13. Nogues J, Schuller IK Exchange bias. Journal of Magnetism and Magnetic Materials. 1999;192(2):203-232.

14. Skumryev V, Stoyanov S, Zhang Y et al. Beating the superparamagnetic limit with exchange bias. Nature. 2003;423(6942):850-853.

15. Lin AL, Peng $\mathrm{H}$, Liu $\mathrm{Z}$ et al. Room temperature magnetic graphene oxide-iron oxide nanocomposite based magnetoresistive random access memory devices via spin-dependent trapping of electrons. Small. 2014;10(10):1945-1952.

16. Colvin VL, Schlamp MC, Alivisatos AP Light-emitting diodes made from cadmium selenide nanocrystals and a semiconducting polymer. Nature. 1994;370:354-357.

17. Novoselov KS, Geim AK, Morozov SV et al. Two-dimensional gas of massless Dirac fermions in graphene. Nature. 2005;438(7065):197-200.

18. Lubbe AS, Alexiou C, Bergemann C Clinical applications of magnetic drug targeting. J Surg Res. 2001;95(2):200-206.

19. Paciotti GF, Myer L, Weinreich D et al. Colloidal gold: a novel nanoparticle vector for tumor directed drug delivery. Drug Deliv. 2004;11(3):169-183.

20. Shenton W, Davis SA, Mann S Directed self-assembly of nanoparticles into macroscopic materials using antibody-antigen recognition. Advanced Materials. 1999;11(6):449-452.

21. Dharmatti R, Phadke C, Mewada A et al. Surface orchestration of gold nanoparticles using cysteamine as linker and folate as navigating molecule for synaphic delivery of doxorubicin. J Nanomed Res. 2014;1(1):00002.

22. Wahajuddi SA, Arora S Superparamagnetic iron oxide nanoparticles: magnetic nanoplatforms as drug carriers. Int $J$ Nanomedicine. 2012;7:3445-3471.

23. Chertok B, Moffat BA, David AE et al. Iron oxide nanoparticles as a drug delivery vehicle for MRI monitored magnetic targeting of brain tumors. Biomaterials. 2008;29(4):487-496.

24. Thiesen B, Jordan A Clinical applications of magnetic nanoparticles for hyperthermia. Int J Hyperthermia. 2008;24(6):467-474.

25. Liz-Marzan LM Tailoring surface plasmons through the morphology and assembly of metal nanoparticles. Langmuir. 2006;22(1):32-41.

26. Lu Y, Liu GL, Lee LP High-density silver nanoparticle film with temperature-controllable interparticle spacing for a tunable surface enhanced Raman scattering substrate. Nano Lett. 2005;5(1):5-9.

27. Xu H, Bjerneld EJ, Kall M et al. Spectroscopy of single hemoglobin molecules by surface enhanced Raman scattering. Phys Rev Lett. 1999;83:4357.

28. Cao YC, Jin R, Mirkin CA Nanoparticles with Raman spectroscopic fingerprints for DNA and RNA detection. Science. 2002;297(5586):1536-1540. 
29. Alvarez-Puebla RA, Agarwal A, Manna P et al. Gold nanorods 3Dsupercrystals as surface enhanced Raman scattering spectroscopy substrates for the rapid detection of scrambled prions. Proc Natl Acad Sci USA. 2011;108(20):8157-8161.

30. Huang X, Jain PK, El-Sayed IH et al. Plasmonic photo thermal therapy (PPTT) using gold nanoparticles. Lasers Med Sci. 2008;23(3):217-228.

31. Rai M, Yadav A, Gade A Silver nanoparticles as a new generation of antimicrobials. Biotechnology Advances. 2009;27(1):76-83.

32. Panacek A, Kolar M, Vecerova $R$ et al. Antifungalactivity of silver nanoparticles against Candida spp. Biomaterials. 2009;30(31):6333-6340.

33. Elechiguerra JL, Burt JL, Morones JR et al. Interaction of silver nanoparticles with HIV-1. J Nanobiotechnology. 2005;3(6):1-10.

34. Tian J, Wong KK, Ho CM et al. Topical delivery of silver nanoparticles promotes wound healing. ChemMedChem. 2007;2(1):129-136.
35. Hendi A Silver nanoparticles mediate differential responses in some of liver and kidney functions during skin wound healing. Journal of King Saud University-Science. 2011;23(1):47-52

36. Alon N, Miroshnikov $\mathrm{Y}$, Perkas $\mathrm{N}$ et al. Substrates coated with silver nanoparticles as a neuronal regenerative material. Int $J$ Nanomedicine. 2014;9(Suppl 1):23-31.

37. AshaRani PV, Low Kah Mun G, Hande MP, Valiyaveettil S (2009) Cytotoxicity and genotoxicity of silver nanoparticles in human cells. ACS Nano 3(2): 279-290.

38. Asharani PV, Wu YL, Gong Z, Valiyaveettil S . Toxicity of silver nanoparticles in zebrafish models. Nanotechnology. 2008;19(25):255102.

39. Johari SA Toxicity Effect of colloidal silver nanoparticles on fertilization capacity and reproduction success of rainbow trout (Oncorhynchus mykiss). J Nanomed Res. 2014;1(1):00001. 